\title{
Electron Microscopy to Probe Flat Band Topological Systems of 2D and Pseudo 2D Quantum Materials
}

David Bell ${ }^{1}$, Joe Checkelsky ${ }^{2}$, Yumbo $\mathrm{Ou}^{2}$, Jagadeesh Moodera ${ }^{2}$, Riccardo Comin ${ }^{2}$ and Aravind Devarakonda $^{2}$

${ }^{1}$ Harvard University, Cambridge, Massachusetts, United States, ${ }^{2}$ Massachusetts Institute of Technology, Cambridge, Massachusetts, United States

Quantum Materials may act as conductors, insulators, semiconductors or even as superconductors. Especially, combinations of different quantum materials are of high interest to explore new phenomena and to build the foundation for future electronic devices at the nanometer scale. The properties of quantum materials differ strongly from the properties of their three-dimensional bulk state. Our research on quantum materials is widely spread, reaching from defect formation in graphene to the characterization of hybrid quantum materials. We use low voltage aberration corrected electron microscopy to investigate chemical various quantum materials. In particular, we will present imaging and analysis of vapor deposition (CVD) graphene with added copper and mercury defects; graphene based hybrid structures, and FeSn Topological Insulators.

The exploration and synthesis constitute only one aspect of the challenges in the development of new topological materials, another challenge is their characterization. Since the phenomena appear at very restricted and dedicated conditions, the characterization method must have very high sensitivity, resolution, localization and precision. The analysis of quantum materials presents new challenges on how to minimize surface and sample damage while imaging and analyzing structures at or beyond the direct atomic level, new approaches are considered in order to correlate materials properties with structure. Some of our multi modal approaches will be discussed in this presentation. As an example we show that the kagome lattice is a two-dimensional network of corner-sharing triangles that is known to host exotic quantum magnetic states (Fig. 1, 2). Theoretical work has predicted that kagome lattices may also host Dirac electronic states that could lead to topological and Chern insulating phases, but these states have so far not been detected in experiments. Here we study the d-electron kagome metal $\mathrm{Fe}_{3} \mathrm{Sn}_{2}$, which is designed to support bulk massive Dirac fermions in the presence of ferromagnetic order.

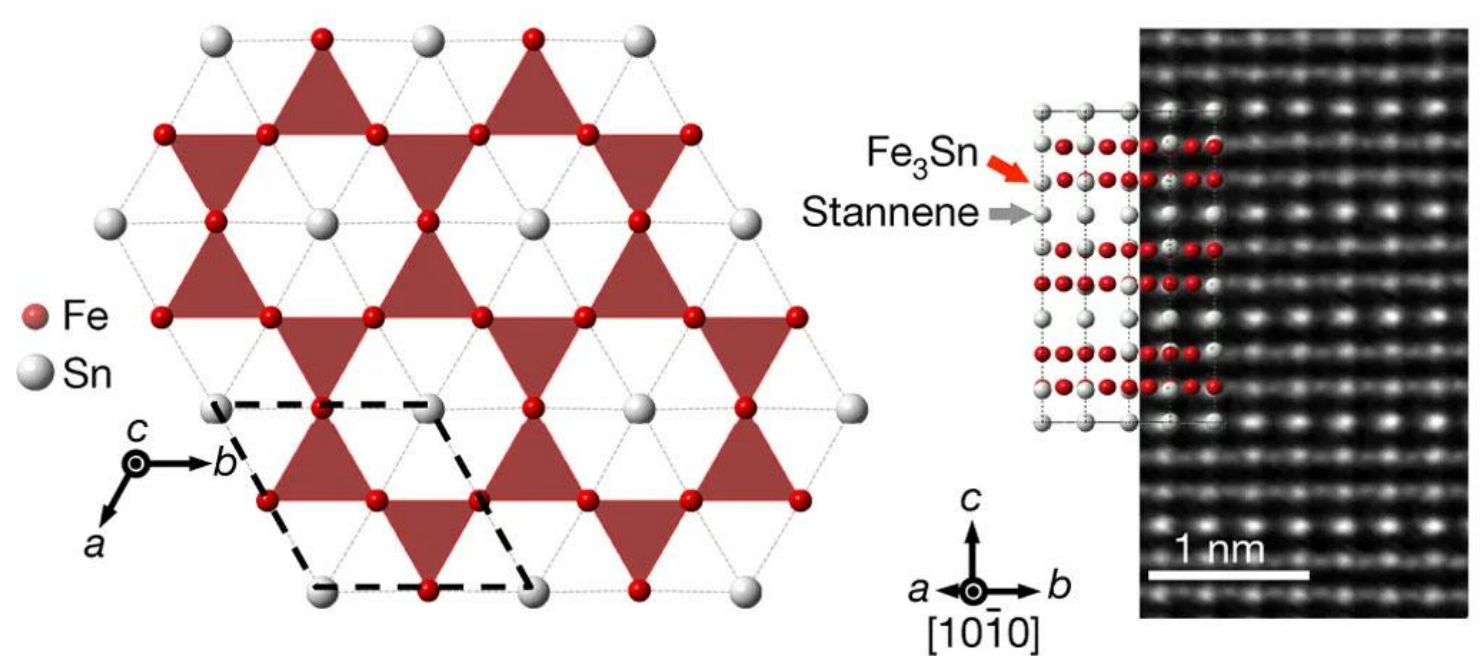


Figure 1. Left, The Fe3Sn kagome plane in Fe3Sn2, with the kagome network shown in red. Right, HADF cross-section of Fe3Sn2 and the corresponding Fe3Sn and stanene layers viewed from the [10-10] direction.

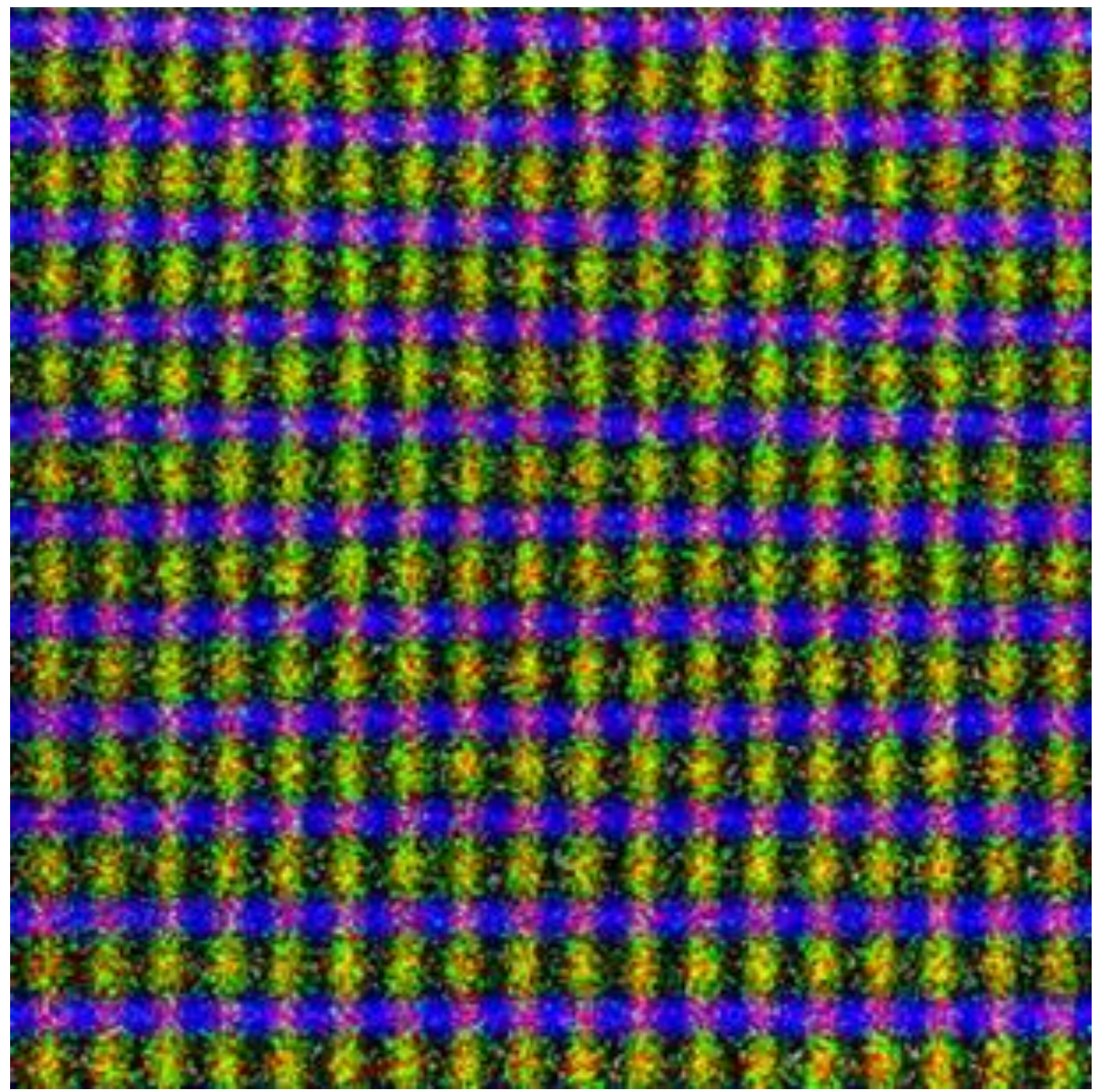

Figure 2. Atomic resolution EDS map of the Co3Sn2S2 again indicating a Kagome structure but clearly indicating the distinct atomic separation of the lattice plains. (With great thanks to Dr. Onishi JEOL Ltd. Japan)

\section{References}

1. Kang et. al., Nature Materials 19, 163-169 (2019).

2. Ye, M. Kang, J. Liu, F. von Cube, C. R. Wicker, T. Suzuki, C Jozwiak, A. Bostwick, E. Rotenberg, D. C. Bell, L. Fu, R. Comin, and J. G. Checkelsky, Nature 555, 638-642 March (2018).

3. This work was supported by the STC Center for Integrated Quantum Materials, NSF Grant No. DMR1231319. 